\title{
Complete $k$-ary trees and generalized meta-Fibonacci sequences
}

\author{
Chris Deugau ${ }^{1}$ and Frank Ruskey ${ }^{1} \|^{\dagger}$ \\ ${ }^{1}$ Dept. of Computer Science, University of Victoria, CANADA
}

We show that a family of generalized meta-Fibonacci sequences arise when counting the number of leaves at the largest level in certain infinite sequences of $k$-ary trees and restricted compositions of an integer. For this family of generalized meta-Fibonacci sequences and two families of related sequences we derive ordinary generating functions and recurrence relations.

Keywords: Meta-Fibonacci sequence, $k$-ary tree, recurrence relation, generating function, ruler function.

\section{Introduction}

A meta-Fibonacci recurrence relation is one of the form

$$
a(n)=a(x(n)-a(n-1))+a(y(n)-a(n-2)),
$$

where $x(n)$ and $y(n)$ are certain linear functions. These recurrence relations have been investigated by several authors in recent years, but their general behavior remains rather mysterious (e.g., Guy [7][Problem E31], Pinn [13]). Perhaps the most well-behaved sequences in the family occur when $x(n)=n$ and $y(n)=n-1$. For a given parameter $s \geq 0$, Jackson and Ruskey [9] showed that the sequences with $x(n)=y(n)+1=n-s$ for $s \geq 0$ are almost as well-behaved. The case of $s=1$ was studied before by Tanny [14]. The case of $s=0$ was considered before by Conolly [4].

Prior to the paper [9], no combinatorial interpretation was known for these sequences (i.e., they were not known to be the solution to some natural counting problem), nor were their generating functions known. The combinatorial interpretation given in [9] was based on binary trees. This paper extends the results of [9] to $k$-ary trees.

We will refer to the sequences $(a(1), a(2), \ldots)$ of solutions to the recurrence relation

$$
a(n)=\sum_{i=1}^{k} a(n-i-(s-1)-a(n-i))
$$

as generalized meta-Fibonacci sequences. These sequences were studied recently by Callaghan, Chew, and Tanny [2], building on the earlier work of Higham and Tanny [8]. In particular, our sequence $a_{0,3}(n)$ is the same as the sequence $T_{0,3}(n+2)$ of [2] for $n \geq 1$ (e.g., Figure 1.5, pg.797). Generalized meta-Fibonacci sequences are quite sensitive to the initial conditions.

We will show that, with the appropriate initial conditions, these sequences also occur in a natural combinatorial setting, that they satisfy a recurrence relation of the form $a(n)=f_{s, k}(n)+a\left(n-g_{s, k}(n)\right)$, and that they have a fairly elegant ordinary generating function. In particular, for any fixed $s \geq 0$ and $k \geq 2$, we give new ways of interpreting the sequences; our interpretations are based on certain subtrees of unusually labelled infinite $k$-ary trees and on certain restricted compositions of an integer. From the combinatorial interpretation, we can easily see that $a(n)$ is monotone, that its consecutive terms increase by 0 or 1 , and that therefore, the sequence hits every positive integer.

\section{Meta-Fibonacci Sequences and Complete $k$-ary Trees}

Figure 1 shows part of an infinite ordered ternary tree $\mathcal{F}_{0,3}$. A $k$-ary version of this tree, $\mathcal{F}_{0, k}$ is defined in the natural way. The forest of labelled trees in $\mathcal{F}_{0, k}$ consists of a succession of complete $k$-ary trees of sizes

${ }^{\dagger}$ Research supported in part by NSERC. 


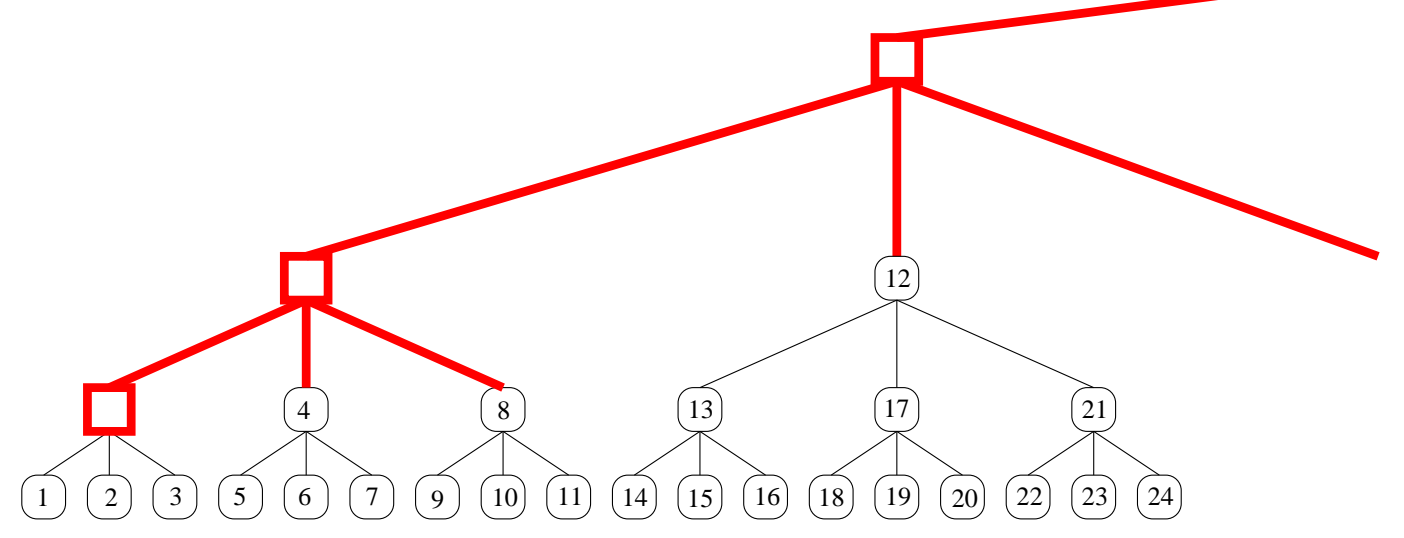

Fig. 1: The tree $\mathcal{F}_{0,3}$.

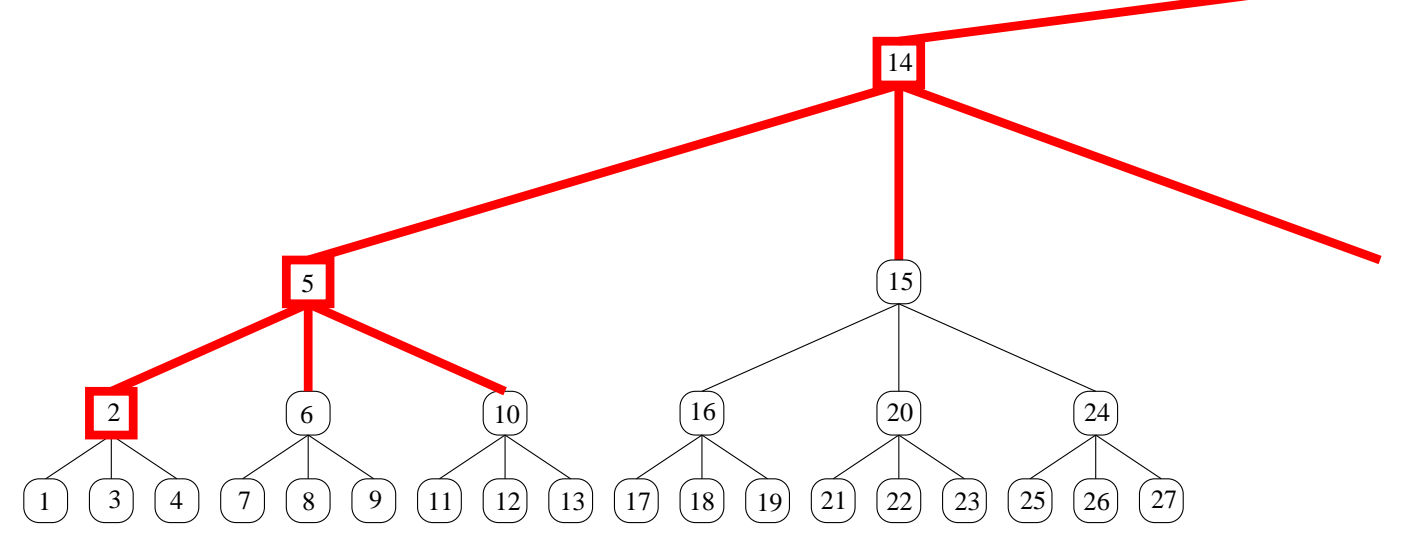

Fig. 2: The tree $\mathcal{F}_{1,3}$.

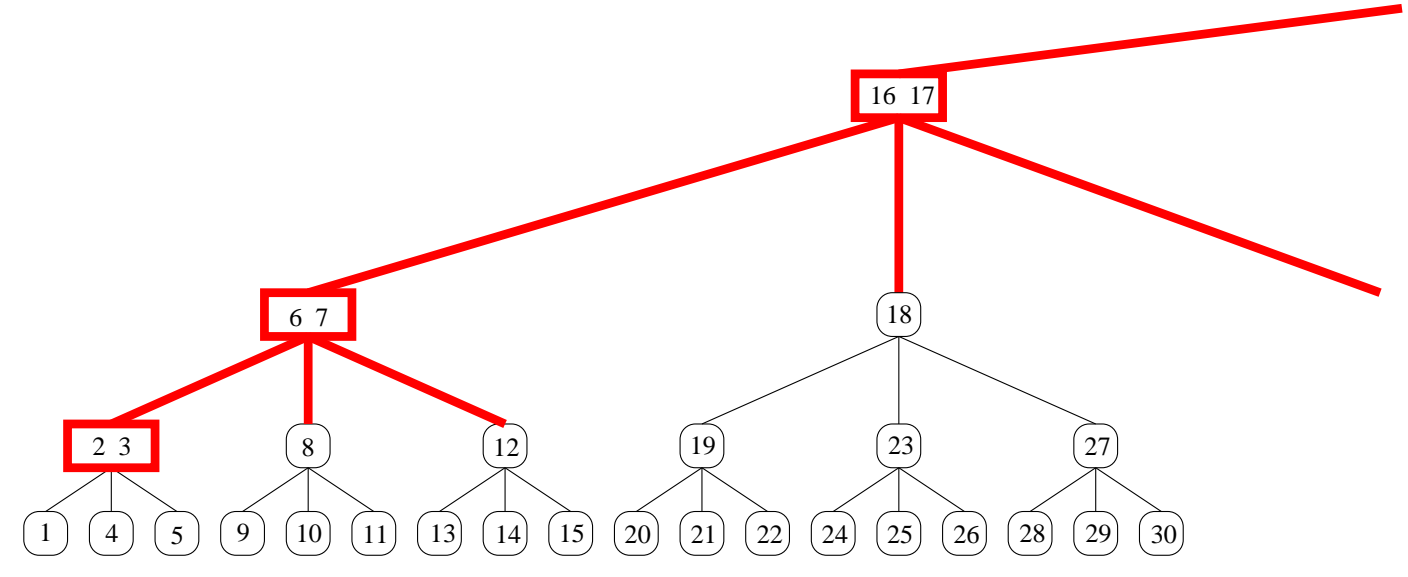

Fig. 3: The tree $\mathcal{F}_{2,3}$. 


\begin{tabular}{c|ccccccccccccccccccc}
$n$ & 1 & 2 & 3 & 4 & 5 & 6 & 7 & 8 & 9 & 10 & 11 & 12 & 13 & 14 & 15 & 16 & 17 & 18 & 19 \\
\hline$a_{0,2}(n)$ & 1 & 2 & 2 & 3 & 4 & 4 & 4 & 5 & 6 & 6 & 7 & 8 & 8 & 8 & 8 & 9 & 10 & 10 & 11 \\
$a_{1,2}(n)$ & 1 & 1 & 2 & 2 & 2 & 3 & 4 & 4 & 4 & 4 & 5 & 6 & 6 & 7 & 8 & 8 & 8 & 8 & 8 \\
$a_{2,2}(n)$ & 1 & 1 & 1 & 2 & 2 & 2 & 2 & 3 & 4 & 4 & 4 & 4 & 4 & 5 & 6 & 6 & 7 & 8 & 8 \\
\hline$d_{0,2}(n)$ & 1 & 1 & 0 & 1 & 1 & 0 & 0 & 1 & 1 & 0 & 1 & 1 & 0 & 0 & 0 & 1 & 1 & 0 & 1 \\
$d_{1,2}(n)$ & 1 & 0 & 1 & 0 & 0 & 1 & 1 & 0 & 0 & 0 & 1 & 1 & 0 & 1 & 1 & 0 & 0 & 0 & 0 \\
$d_{2,2}(n)$ & 1 & 0 & 0 & 1 & 0 & 0 & 0 & 1 & 1 & 0 & 0 & 0 & 0 & 1 & 1 & 0 & 1 & 1 & 0 \\
\hline$p_{0,2}(n)$ & 1 & 2 & 4 & 5 & 8 & 9 & 11 & 12 & 16 & 17 & 19 & 20 & 23 & 24 & 26 & 27 & 32 & 33 & 35 \\
$p_{1,2}(n)$ & 1 & 3 & 6 & 7 & 11 & 12 & 14 & 15 & 20 & 21 & 23 & 24 & 27 & 28 & 30 & 31 & 37 & 38 & 40 \\
$p_{2,2}(n)$ & 1 & 4 & 8 & 9 & 14 & 15 & 17 & 18 & 24 & 25 & 27 & 28 & 31 & 32 & 34 & 35 & 42 & 43 & 45 \\
\hline \hline$a_{0,3}(n)$ & 1 & 2 & 3 & 3 & 4 & 5 & 6 & 6 & 7 & 8 & 9 & 9 & 9 & 10 & 11 & 12 & 12 & 13 & 14 \\
$a_{1,3}(n)$ & 1 & 1 & 2 & 3 & 3 & 3 & 4 & 5 & 6 & 6 & 7 & 8 & 9 & 9 & 9 & 9 & 10 & 11 & 12 \\
$a_{2,3}(n)$ & 1 & 1 & 1 & 2 & 3 & 3 & 3 & 3 & 4 & 5 & 6 & 6 & 7 & 8 & 9 & 9 & 9 & 9 & 9 \\
\hline$d_{0,3}(n)$ & 1 & 1 & 1 & 0 & 1 & 1 & 1 & 0 & 1 & 1 & 1 & 0 & 0 & 1 & 1 & 1 & 0 & 1 & 1 \\
$d_{1,3}(n)$ & 1 & 0 & 1 & 1 & 0 & 0 & 1 & 1 & 1 & 0 & 1 & 1 & 1 & 0 & 0 & 0 & 1 & 1 & 1 \\
$d_{2,3}(n)$ & 1 & 0 & 0 & 1 & 1 & 0 & 0 & 0 & 1 & 1 & 1 & 0 & 1 & 1 & 1 & 0 & 0 & 0 & 0 \\
\hline$p_{0,3}(n)$ & 1 & 2 & 3 & 5 & 6 & 7 & 9 & 10 & 11 & 14 & 15 & 16 & 18 & 19 & 20 & 22 & 23 & 24 & 27 \\
$p_{1,3}(n)$ & 1 & 3 & 4 & 7 & 8 & 9 & 11 & 12 & 13 & 17 & 18 & 19 & 21 & 22 & 23 & 25 & 26 & 27 & 30 \\
$p_{2,3}(n)$ & 1 & 4 & 5 & 9 & 10 & 11 & 13 & 14 & 15 & 20 & 21 & 22 & 24 & 25 & 26 & 28 & 29 & 30 & 33
\end{tabular}

Tab. 1: The values of $a_{s, k}(n), d_{s, k}(n)$, and $p_{s, k}(n)$ for $k=2,3, s=0,1,2$, and $1 \leq n \leq 19$.

$$
1, \underbrace{1,1, \ldots, 1}_{k-1}, \underbrace{1+k, \ldots, 1+k}_{k-1}, \ldots, \underbrace{1+k+\cdots+k^{h}, \ldots, 1+k+\cdots+k^{h}}_{k-1}, \ldots
$$

The nodes of these subtrees are labelled in preorder. In $\mathcal{F}_{0, k}$ there is a one-way infinite path of unlabelled nodes (drawn with rectangles in Figure 11, which we refer to as the delay path. The rightmost $(k-1)$ subtrees of delay node $h$ are referred to as subforest $h$, where $h \in\{0,1,2, \ldots\}$ is the common height of the trees in the subforest. For instance, the first super-node is the parent of subforest 1 , the second super-node is the parent of subforest 2 , and so on. We will now generalize to $\mathcal{F}_{s, k}$. The structure of the tree is the same as for $\mathcal{F}_{0, k}$; it is only the labelling of the delay nodes that changes. The trees $\mathcal{F}_{1,3}$ and $\mathcal{F}_{2,3}$ are shown in Figures 2 and 3 Except along the delay path, each subtree is again labelled in preorder. The delay path is parameterized by a value $s$ that gives the delay between the preorder counts of successive trees, where the delay is applied after the leftmost subtree of a given size. Alternatively, we can think of the nodes along the path as being super-nodes, where each super-node contains $s$ ordinary nodes. Note that the nodes of the delay path occur at the positions between the underbraces in (1).

Denote by $\mathcal{T}_{s, k}(n)$ the tree induced by the first $n$ labelled nodes of the infinite tree $\mathcal{F}_{s, k}$. Define $a_{s, k}(n)$ to be the number of nodes at the bottom level in $\mathcal{T}_{s, k}(n)$. Also define $d_{s, k}(n)$ to be 1 if the $n$-th node is a leaf and to be 0 if the $n$-th node is an internal node. Finally, define $p_{s, k}(n)$ to be the positions occupied by the 1 's in the $d_{s, k}(n)$ sequence. Since $\mathcal{T}_{s, k}(0)$ is an empty tree, it has no nodes at the bottom level. Therefore, $a_{s, k}(0)=0$, which implies $d_{s, k}(0)=p_{s, k}(0)=0$. Table 11 gives the values of $a_{s, k}(n)$, $d_{s, k}(n)$, and $p_{s, k}(n)$ for $k=2,3, s=0,1,2$ and $1 \leq n \leq 20$. The values of four of these table entries appear in OEIS ${ }^{(\mathrm{i})}$, namely $a_{0,2}(n)=A 046699, a_{1,2}(n)=A 006949, d_{0,2}(n)=A 079559$, and $p_{0,2}(n)=A 101925=A 005187(n)+1$. For fixed $s$ these numbers are related as follows,

$$
a_{s, k}(n)=\sum_{j=0}^{n} d_{s, k}(j) \text { and } p_{s, k}(n)=\min \left\{j: a_{s, k}(j)=n\right\} .
$$

In the sequel we will drop the $s, k$ subscripts, since our discussion will be for fixed values of these parameters, and it will make the notation less cumbersome.

Theorem 2.1 If $1 \leq n \leq s+1$, then $a(n)=1$. If $n=s+i$ and $2 \leq i \leq k$ then $a(n)=i$. If $n>s+k$, then

$$
a(n)=\sum_{i=1}^{k} a(n-i-(s-1)-a(n-i)) .
$$

\footnotetext{
(i) OEIS = Neil Sloane's online encyclopedia of integer sequences.
} 
Proof: First observe that if all the leaves at the last level are removed from $\mathcal{F}_{s, k}$, then the same structure remains, once the leftmost super-node is made into an ordinary node (by subtracting $s-1$ ). We will refer to this process as chopping the last level. The number of nodes at the penultimate level of $\mathcal{T}_{s, k}(n)$ can be obtained by chopping the last level of the tree, and then counting how many nodes are at the bottom level of a tree containing that same number of nodes. The number of nodes in the chopped tree is $n-(s-1)-a(n)$. Therefore, the number of nodes at the penultimate level of $\mathcal{T}_{s, k}(n)$ (counting the super-node only once) is

$$
a(n-(s-1)-a(n)) .
$$

Also observe that if each node at the penultimate level of $\mathcal{T}_{s, k}(n)$ has $k$ children, then the number of nodes at the penultimate level is $a(n) / k$. However, the rightmost node on the penultimate level will not necessarily have $k$ children. Assume that the rightmost node on the penultimate level has $r$ children. If we add $k-r$ nodes to the bottom level of $\mathcal{T}_{s, k}(n)$, then the rightmost node has $k$ children, and we can divide by $k$ to determine the number of nodes at the penultimate level. Therefore, another expression for the number of nodes at the penultimate level of $\mathcal{T}_{s, k}(n)$ is

$$
(a(n)+k-r) / k .
$$

Finally, observe that if the rightmost node at the penultimate level has $r$ children, and we subtract $r$ nodes from the bottom level of $\mathcal{T}_{s, k}(n)$, then the rightmost node at the penultimate level has 0 children. If we divide by $k$, we would be counting every node at the penultimate level other than the rightmost node. Therefore, an expression for the number of nodes at the penultimate level, other than the rightmost node, of $\mathcal{T}_{s, k}(n)$ is

$$
(a(n)-r) / k
$$

We split the proof into two broad cases depending on whether $n$ is a leaf or not; i.e., whether $d(n)=1$ (Case 1) or $d(n)=0$ (Case 2). In either case, we will be computing $a(n)$, the number of nodes at the bottom level of our tree $\mathcal{T}_{s, k}(n)$, by counting each node $p$ that is at the penultimate level of our tree $p_{c}$ times, where $p_{c}$ is the number of children of node $p$.

Case 1: If $d(n)=1$, then node $n$ is the $r$ th child of node $n-r$. Each of the $r$ trees

$\mathcal{T}_{s, k}(n-1), \mathcal{T}_{s, k}(n-2), \ldots, \mathcal{T}_{s, k}(n-r)$ have node $n-r$ at the penultimate level, and therefore each has $(a(n)+k-r) / k$ nodes at the penultimate level. Each of the $k-r$ remaining trees

$\mathcal{T}_{s, k}(n-r-1), \mathcal{T}_{s, k}(n-r-2), \cdots, \mathcal{T}_{s, k}(n-k)$ does not have $n-r$ at the penultimate level, and therefore has $(a(n)-r) / k$ nodes at the penultimate level. Recall that for any $m$, the tree $\mathcal{T}_{s, k}(m)$ has $a(m-(s-1)-a(m))$ nodes at the penultimate level. Thus

$$
\begin{aligned}
\sum_{i=1}^{k} a(n-i-(s-1)-a(n-i)) & \\
& =\sum_{i=1}^{r} a(n-i-(s-1)-a(n-i))+\sum_{i=r+1}^{k} a(n-i-(s-1)-a(n-i)) \\
& =\sum_{m=n-r}^{n-1} a(m-(s-1)-a(m))+\sum_{m=n-k}^{n-(r+1)} a(m-(s-1)-a(m)) \\
& =\sum_{m=n-r}^{n-1}(a(n)+k-r) / k+\sum_{m=n-k}^{n-(r+1)}(a(n)-r) / k \\
& =r(a(n)+k-r) / k+(k-r)(a(n)-r) / k \\
& =(r a(n)+r k-r 2+k a(n)-k r-r a(n)+r 2) / k \\
& =k a(n) / k \\
& =a(n) .
\end{aligned}
$$

Case 2: Omitted for space reasons. Will be included with the full paper.

Define $\mathcal{D}_{s, k}$ to be the infinite string $d_{s, k}(1) d_{s, k}(2) d_{s, k}(3) \cdots$. Let $D_{n, k}$ be the finite string defined by $D_{0, k}=1$ and $D_{n+1, k}=0\left(D_{n, k}\right)^{k}$, the string with 0 at the start, followed by $k$ repetitions of $D_{n, k}$. Let $E_{n, k}$ be the finite string defined by $E_{0, k}=1$ and $E_{n+1, k}=\left(E_{n, k}\right)^{k} 0$, the string starting with $k$ repetitions of $E_{n, k}$ followed by 0 . As before, we will drop the subscripts $s$ and/or $k$ when no confusion can arise. 
Lemma 2.2 For all $n \geq 0$, we have $0^{n} E_{n}=D_{n} 0^{n}$.

Proof: Our proof is by induction on $n$. The statement is true if $n=0$ since $D_{0}=E_{0}=1$. Assuming that it is true for $n$, for $n+1$ we have

$$
0^{n+1} E_{n+1}=00^{n}\left(E_{n}\right)^{k} 0=0\left(D_{n}\right)^{k} 0^{n} 0=D_{n+1} 0^{n+1} .
$$

Lemma 2.3 For $n \geq 0$ and $k \geq 2$,

$$
D_{0}\left(D_{0}\right)^{k-1}\left(D_{1}\right)^{k-1} \cdots\left(D_{n}\right)^{k-1}=\left(E_{n}\right)^{k-1}\left(E_{n-1}\right)^{k-1} \cdots\left(E_{1}\right)^{k-1}\left(E_{0}\right)^{k-1} E_{0} .
$$

Proof: Our proof is by induction on $n$. If $n=0$, then $\left(D_{0}\right)^{k}=\left(E_{0}\right)^{k}=1^{k}$. For the general case we will first prove, also by induction on $n$, that

$$
\left(D_{n}\right)^{k-1}=0^{n}\left(E_{n}\right)^{k-2}\left(E_{n-1}\right)^{k-1} \ldots\left(E_{1}\right)^{k-1}\left(E_{0}\right)^{k-1} E_{0}
$$

Equation (8) is true if $n=0$. For $n+1$ we have

$$
\begin{aligned}
\left(D_{n+1}\right)^{k-1} & =\left(D_{n+1}\right)^{k-2} D_{n+1} \\
& =\left(D_{n+1}\right)^{k-2} 0\left(D_{n}\right)^{k} \\
& =\left(D_{n+1}\right)^{k-2} 0 D_{n}\left(D_{n}\right)^{k-1} \\
& =\left(D_{n+1}\right)^{k-2} 0 D_{n} 0^{n}\left(E_{n}\right)^{k-2}\left(E_{n-1}\right)^{k-1} \cdots\left(E_{1}\right)^{k-1}\left(E_{0}\right)^{k-1} E_{0} \\
& =\left(D_{n+1}\right)^{k-2} 0^{n+1} E_{n}\left(E_{n}\right)^{k-2}\left(E_{n-1}\right)^{k-1} \ldots\left(E_{1}\right)^{k-1}\left(E_{0}\right)^{k-1} E_{0} \\
& =0^{n+1}\left(E_{n+1}\right)^{k-2}\left(E_{n}\right)^{k-1}\left(E_{n-1}\right)^{k-1} \cdots\left(E_{1}\right)^{k-1}\left(E_{0}\right)^{k-1} E_{0} .
\end{aligned}
$$

In a somewhat similar fashion we may also prove by induction that

$$
\left(E_{n}\right)^{k-1}\left(E_{n-1}\right)^{k-1} \ldots\left(E_{1}\right)^{k-1}\left(E_{0}\right)^{k-1} E_{0} 0^{n+1}=E_{n+1} .
$$

Now back to the proof of the lemma. Assuming that it is true for $n$, then for $n+1$

$$
\begin{aligned}
D_{0} & \left(D_{0}\right)^{k-1}\left(D_{1}\right)^{k-1} \cdots\left(D_{n}\right)^{k-1}\left(D_{n+1}\right)^{k-1} \\
& =\left(E_{n}\right)^{k-1}\left(E_{n-1}\right)^{k-1} \cdots\left(E_{1}\right)^{k-1}\left(E_{0}\right)^{k}\left(D_{n+1}\right)^{k-1} \\
& =\left(E_{n}\right)^{k-1}\left(E_{n-1}\right)^{k-1} \cdots\left(E_{1}\right)^{k-1}\left(E_{0}\right)^{k} 0^{n+1}\left(E_{n+1}\right)^{k-2}\left(E_{n}\right)^{k-1} \cdots\left(E_{1}\right)^{k-1}\left(E_{0}\right)^{k-1} E_{0} \\
& =E_{n+1}\left(E_{n+1}\right)^{k-2}\left(E_{n}\right)^{k-1} \cdots\left(E_{1}\right)^{k-1}\left(E_{0}\right)^{k-1} E_{0} \\
& =\left(E_{n+1}\right)^{k-1}\left(E_{n}\right)^{k-1} \cdots\left(E_{1}\right)^{k-1}\left(E_{0}\right)^{k} .
\end{aligned}
$$

The first equality follows from the inductive assumption; the second follows from (8); the third follows from (9).

\section{Lemma 2.4}

$$
\mathcal{D}_{0, k}=D_{0}\left(D_{0}\right)^{k-1}\left(D_{1}\right)^{k-1}\left(D_{2}\right)^{k-1}\left(D_{3}\right)^{k-1} \cdots=E_{\infty}
$$

Proof: The first equality in 10 is implied immediately by the definition of $\mathcal{F}_{0, k}$; i.e., in $0 D_{n} D_{n} \cdots$ the 0 is from the root (which is listed first in preorder) and $D_{n} D_{n} \cdots$ are the subtrees of height $n$. Since there will be $k-1$ extra subtrees of height $n+1$, (one subtree has already been defined), we need to make sure to repeat this substring $k-1$ times.

The second equality comes from Lemma 2.3 . Since $E_{n}$ is a prefix of $E_{n+1}$, the expression $E_{\infty}$ is welldefined. Hence $\mathcal{D}_{0, k}=E_{\infty}$.

In the notation for the $q$-binomial coefficients [3], we have $\left[\begin{array}{l}h \\ 1\end{array}\right]_{k}=1+k+\cdots+k^{h-1}=\frac{k^{h}-1}{k-1}$. In this paper, the bottom term will always be one, so we will use the notation $[h]_{k}$ to represent $\left[\begin{array}{l}h \\ 1\end{array}\right]_{k}$. When no confusion can arise the subscript $k$ will be dropped. 
Lemma 2.5 For $n \geq 0$, we have $\left|D_{n}\right|=\left|E_{n}\right|=[h+1]$.

Proof: It is obvious that $\left|D_{n}\right|=\left|E_{n}\right|$. We know that $D_{0}=1$, so $\left|D_{0}\right|=1=[0+1]$. Since $D_{n+1}=$ $0\left(D_{n}\right)^{k}$, inductively, $\left|D_{n+1}^{k}\right|=1+k[n+1]=1+k\left(1+k+\cdots+k^{n}\right)=[n+2]$.

Corollary 2.6 The numbers $a_{0, k}(n)$ satisfy the following recurrence relation for $0 \leq m<k^{h}$.

$$
a_{0, k}([h]+m)=k^{h-1}+a_{0, k}(m) .
$$

Proof: Since $\mathcal{D}_{0}=E_{h} E_{h} \cdots=\left(E_{h-1}\right)^{k} 0\left(E_{h-1}\right)^{k} 0\left(E_{h-1}\right)^{k} 0 \cdots$ and $\left|E_{h-1}\right|=[h]$, the equality $d([h]+$ $m)=d(m)$ holds for $1 \leq m \leq k^{h}-1$. The range for $m$ comes from the fact that $\left|\left(E_{h-1}\right)^{k}\right|=[h]+(k-$ 1) $[h]=[h]+k^{h}-1$. Since we defined $d(0)=0$ the range can be extended to include $m=0$. The number of 1 's in $E_{h-1}$ is $\#_{1}\left(E_{h-1}\right)=k^{h-1}$. Thus

$$
a_{0, k}([h]+m)=\sum_{p=0}^{[h]} d(p)+\sum_{p=0}^{m} d([h]+p)=\#_{1}\left(E_{h-1}\right)+\sum_{p=0}^{m} d_{0}(p)=k^{h-1}+a_{0, k}(m) .
$$

Lemma 2.7 For $s \geq 0$ and $k \geq 2$,

$$
a_{s, k}(n)= \begin{cases}a_{0, k}(n-s h) & \text { if }[h]+(s-1) h+2 \leq n \leq[h+1]+(s-1) h, \\ k^{h-1} & \text { if }[h]+(s-1) h-s+2 \leq n \leq[h]+(s-1) h+1 .\end{cases}
$$

Proof: The labels on the nodes in subforest $h$ in $\mathcal{F}_{s, k}$ are exactly the values of $n$ lying in the first range above. This is true because the number of ordinary nodes to the left of $h$ can be found by adding the number of nodes in all of the subtrees. The first subtree is simply one node. The remaining subtrees are $k$-ary trees of height $j=1,2, \ldots, h-1$. These $k$-ary trees each have $1+k+\cdots+k^{j-1}=[j]$ nodes. By the construction of $\mathcal{F}_{s, k}$, we have $k-1$ of each subtree of height $j$ (except, as previously mentioned, the extra tree of height 1). Summing the number of nodes in all of the subtrees gives us

$$
1+\sum_{j=1}^{h-1}(k-1)[j]=1+\sum_{j=1}^{h-1}\left(k^{j}-1\right)=-h+2+\sum_{j=1}^{h-1} k^{j}=[h]-h+1 .
$$

The number of super-nodes is $s h$. Thus, the lowest label of a node in subforest $h$ of our tree is $[h]+$ $(s-1) h+1+1=[h]+(s-1) h+2$ and the highest label is $[h]+(s-1) h+1+(k-1)[h]=(s-1) h+$ $1+k\left(1+k+\cdots+k^{h-1}\right)=[h+1]+(s-1) h$.

Corollary $2.8 a_{1, k}(n)=a\left(n-\left\lfloor\log _{k}((n-1)(k-1)+1)\right\rfloor\right)$

Proof: If $s=1$, the super-nodes of $\mathcal{F}_{1, k}$ are numbered $[h]+1$. Hence node $n$ is contained in subforest $h=\left\lfloor\log _{k}((n-1)(k-1)+1)\right\rfloor$.

Taking $s=1$ in Lemma 2.7 we obtain $a_{1, k}(n)=a_{0, k}(n-h)$ in the range $[h]+2 \leq n \leq[h+1]$. In that range $h=\left\lfloor\log _{k}((n-1)(k-1)+1)\right\rfloor$. We need to check what happens when $n$ is a super-node, in other words, when $n=[h]+1$. By the Lemma 2.7 $a_{1, k}([h]+1)=k^{h-1}$. In $\mathcal{F}_{0, k}$, the node $[h]+1-h$ is the rightmost node in subforest $h-1$, and thus $a_{0, k}([h]+1-h)=k^{h-1}$.

Theorem 2.9 If $1 \leq n \leq s+1$, then $a(n)=1$. If $n=s+i$ and $2 \leq i \leq k$ then $a(n)=i$. If $n>s+k$, then If $[h]+(s-1) h-s+2 \leq n \leq[h]+(s-1) h+2$ then

$$
a(n)=k^{h-1}
$$

If $1 \leq m \leq[h-1]$ then

$$
a([h]+(s-1) h+2+m)=k^{h-2}+a([h]+1+(s-1) h+m-[h-1]-s)
$$




$$
\begin{aligned}
& \text { If } 1 \leq m \leq k^{h-1}-1 \text { then } \\
& \qquad \begin{array}{l}
a([h]+[h-1]+(s-1) h+2+m)=k^{h-2}+a([h]+(s-1) h+2+m) \\
\text { If } 1 \leq m \leq(k-2)[h] \text { then } \\
\quad a(2[h]+(s-1) h+1+m)=k^{h-1}+a([h]+(s-1) h+1+m)
\end{array}
\end{aligned}
$$

Proof: Omitted for space reasons. To be included in full paper.

Corollary 2.10 There are functions $f_{s, k}(n)$ and $g_{s, k}(n)$ such that $a(n)=f_{s, k}(n)+a\left(n-g_{s, k}(n)\right)$.

Proof: The existence of such functions should be clear from Theorem 2.9 but finding a nice expression for them is a challenge and is omitted. For example, one of the breaks in the cases above occurs when $n=[h]+(s-1)(h-1)+1$. This can be solved for $h$ to yield

$$
h=\frac{1}{(s-1)(k-1) \ln k}\left((k-1)(n-2+s)+1-(s-1)(k-1) W\left(\frac{k^{((k-1)(n-2+s)+1) /((s-1)(k-1))} \ln k}{(s-1)(k-1)}\right)\right),
$$

where $W$ is the Lambert-W function.

Let $r_{1}, r_{2}, r_{3}, r_{4}, \cdots$ be the transition sequence of the $k$-ary reflected Gray code; in the case of $k=2$ this sequence is also known as the "ruler function" (A001511). The generalized ruler sequence is $R_{\infty}$ where $R_{1}=1^{k-1}$ and $R_{n+1}=\left(R_{n}, n+1\right)^{k-1}, R_{n}$. Observe that if all the 0 's are removed from the sequence $r_{1}-1, r_{2}-1, r_{3}-1, r_{4}-1, \ldots$ then the ruler function is again obtained. The non-zero values occur in the positions that are divisible by $k$.

\section{Lemma 2.11}

$$
\begin{aligned}
\mathcal{D}_{0} & =1^{k} 0^{r_{1}} 1^{k} 0^{r_{2}} 1^{k} 0^{r_{3}} 1^{k} 0^{r_{4}} \ldots \\
& =10^{r_{1}-1} 10^{r_{2}-1} 10^{r_{3}-1} 10^{r_{4}-1} \ldots
\end{aligned}
$$

Proof: Equation $(13)$ is true by the observation made above. Since $\left|R_{n}\right|=k^{n}-1$, we have $r_{k^{n}+i}=r_{i}$ for $1 \leq i \leq k^{n+1}-k^{n}-1$ and $r_{k^{n}}=n+1$. We will show that

$$
E_{n}=1^{k} 0^{r_{1}} 1^{k} 0^{r_{2}} \cdots 1^{k} 0^{r_{k-1}-1},
$$

which will finish the proof of 12 since $\mathcal{D}_{0}=E_{\infty}$ by Lemma 2.4. Since we know for $n=0$ that $E_{1}=E_{0}^{k} 0$, we can proceed by induction and find

$$
\begin{aligned}
& E_{n+1}=\left(E_{n}\right)^{k} 0 \\
& =1^{k} 0^{r_{1}} 1^{k} 0^{r_{2}} \cdots 1^{k} 0^{r_{k} n-1} 1^{k} 0^{r_{1}} 1^{k} 0^{r_{2}} \cdots 1^{k} 0^{r_{k}-1} 0 \\
& =1^{k} 0^{r_{1}} 1^{k} 0^{r_{2}} \cdots 1^{k} 0^{r_{k-1} n} 1^{k} 0^{r_{k-1}{ }^{n-1}} 1^{k} 0^{r_{k^{n-1}+2}} \cdots 1^{k} 0^{r_{k} n-1} 1^{k} 0^{r_{k-1}-1} 0 \\
& =1^{k} 0^{r_{1}} 1^{k} 0^{r_{2}} \cdots 1^{k} 0^{r_{k-1}} 1^{k} 0^{r_{k^{n-1}+1}} 1^{k} 0^{r_{k^{n-1}+2}} \cdots 1^{k} 0^{r_{k}-1} 1^{k} 0^{n+1} \text {. }
\end{aligned}
$$

We can extend some of the previous results about $\mathcal{D}_{0}$ to $\mathcal{D}_{s}$. For proposition $P$ the notation $\llbracket P \rrbracket$ means 1 if $P$ is true and 0 if $P$ is false.

Lemma 2.12 Let $s_{j}=r_{j}+s \llbracket j$ is a power of $k \rrbracket$. Then

$$
\begin{aligned}
& \mathcal{D}_{s}=D_{0} 0^{s}\left(D_{0}\right)^{k-1} 0^{s}\left(D_{1}\right)^{k-1} 0^{s}\left(D_{2}\right)^{k-1} \ldots \\
& \mathcal{D}_{s}=10^{s_{1}-1} 10^{s_{2}-1} 10^{s_{3}-1} 10^{s_{4}-1} \ldots
\end{aligned}
$$


Proof: Equation 15 comes from our construction of $\mathcal{F}_{s, k}$. The $0^{s}$ terms represent where the super-nodes go in our construction, and since $d_{s}(n)=0$ when $n$ is an internal node, we will have $s 0$ 's.

Equation (16) comes from the second equality in Lemma 2.11. and the fact that a new super-node will be added after we have seen a complete left subtree, which will have $k^{i}$ leaf nodes, where $i$ is an integer. Therefore, we need to add $s$ 's after every $k^{i}$-th leaf node, where $i$ is an integer. This gives us

$$
\begin{aligned}
\mathcal{D}_{s} & =10^{s} 0^{r_{1}-1} 10^{r_{2}-1} \cdots 10^{s} 0^{r_{k}-1} 1 \cdots 10^{s} 0^{r_{k 2}-1} \ldots \\
& =10^{s+r_{1}-1} 10^{r_{2}-1} \cdots 10^{s+r_{k}-1} 1 \cdots 10^{s+r_{k 2}-1} \ldots \\
& =10^{s_{1}-1} 10^{s_{2}-1} 10^{s_{3}-1} 10^{s_{4}-1} 10^{s_{5}-1} 10^{s_{6}-1} 10^{s_{7}-1} 10^{s_{8}-1} 10^{s_{9}-1} \ldots
\end{aligned}
$$

Since the $p_{s, k}(n)$ numbers give the positions of the 1 's in $\mathcal{D}_{s}$ the following corollary is true. The $s=1$ case of this corollary is contained in Proposition 4.1 of [8].

Corollary 2.13 For all $n \geq 1$,

$$
p_{s}(n+1)-p_{s}(n)=r_{n}+s \llbracket n \text { is a power of } k \rrbracket .
$$

By summing the equation in Corollary 2.13 we obtain

$$
\begin{aligned}
p_{s}(n) & =1+\epsilon_{k}((n-1) !)+s\left\lceil\log _{k} n\right\rceil \\
& =s\left\lceil\log _{k} n\right\rceil+\sum_{j \geq 0}\left\lfloor\frac{n-1}{k^{j}}\right\rfloor,
\end{aligned}
$$

where $\epsilon_{q}(m)$ denotes the largest power of $q$ that divides $m$ (see [6], pg. 114). For $s=1$, this result is closely related to Proposition 4.1 of [8].

\subsection{Generating Functions}

If $S=s(1) s(2) s(3) \cdots$ is a string then we use $S(z)$ to denote the ordinary generating function $S(z)=$ $\sum_{k \geq 1} s(i) z^{i}$. Let $\mathcal{A}_{s}(z)$ and $\mathcal{D}_{s}(z)$ denote the ordinary generating functions of the $a_{s, k}(n)$ and $d_{s, k}(n)$ sequences, respectively. Directly from the definitions we get the equation shown below:

$$
\mathcal{A}_{s}(z)=\frac{\mathcal{D}_{s}(z)}{1-z}
$$

Since $\mathcal{A}_{s}(z)$ is determined by $\mathcal{D}_{s}(z)$ and $\mathcal{D}_{s}(z)$ is easier to treat, we first concentrate our attention on $\mathcal{D}_{s}(z)$.

\section{Lemma 2.14}

$$
\begin{aligned}
D_{n}(z) & =z^{n+1}\left(1+z^{[1]}+z^{2[1]}+\cdots+z^{(k-1)[1]}\right) \cdots\left(1+z^{[n]}+\cdots+z^{(k-1)[n]}\right) \\
& =z^{n+1} \prod_{i=1}^{n} \sum_{j=0}^{k-1} z^{j[i]}=z^{n+1} \prod_{i=1}^{n} \frac{1-z^{k[i]}}{1-z^{[i]}} \\
E_{n}(z) & =z\left(1+z^{[1]}+z^{2[1]}+\cdots+z^{(k-1)[1]}\right) \cdots\left(1+z^{[n]}+\cdots+z^{(k-1)[n]}\right) \\
& =z \prod_{i=1}^{n} \sum_{j=0}^{k-1} z^{j[i]}=z \prod_{i=1}^{n} \frac{1-z^{k[i]}}{1-z^{[i]}} .
\end{aligned}
$$

Proof: From the recurrence relation $D_{0}=1$ and $D_{n+1}=0\left(D_{n}\right)^{k}$ we obtain $D_{0}(z)=z$ and

$$
\begin{aligned}
D_{n+1}(z) & =z D_{n}(z)+z^{\left|0 D_{n}\right|} D_{n}(z)+z^{\left|0\left(D_{n}\right)^{2}\right|} D_{n}(z)+\cdots+z^{\left|0\left(D_{n}\right)^{k-1}\right|} D_{n}(z) \\
& =z D_{n}(z)+z^{[n+1]+1} D_{n}(z)+z^{2[n+1]+1} D_{n}(z)+\cdots+z^{(k-1)[n+1]+1} D_{n}(z) \\
& =z\left(1+z^{[n+1]}+z^{2[n+1]}+\cdots+z^{(k-1)[n+1]}\right) D_{n}(z) .
\end{aligned}
$$

Similarly, $E_{0}(z)=z$ and $E_{n+1}(z)=\left(1+z^{[n+1]}+z^{2[n+1]}+\cdots+z^{(k-1)[n+1]}\right) E_{n}(z)$. The results now follow by induction. 
Corollary 2.15

$$
\mathcal{D}_{0}(z)=z \prod_{i \geq 1} \sum_{j=0}^{k-1} z^{j[i]}=z \prod_{i \geq 1}^{n} \cdot \frac{1-z^{k[i]}}{1-z^{[i]}}
$$

Proof: Follows from Lemma 2.14 and the equation $\mathcal{D}_{0}=E_{\infty}$ from Lemma 2.4

Theorem 2.16 The generating function $\mathcal{D}_{s}^{k}(z)$ is equal to

$$
z\left(1+z^{s+k^{0}}\left(\frac{1-z^{(k-1)[1]}}{1-z^{[1]}}+z^{s+k^{1}} \frac{1-z^{k[1]}}{1-z^{[1]}}\left(\frac{1-z^{(k-1)[2]}}{1-z^{[2]}}+z^{s+k^{2}} \frac{1-z^{k[2]}}{1-z^{[2]}}\left(\frac{1-z^{(k-1)[3]}}{1-z^{[3]}} \cdots\right.\right.\right.\right.
$$

Proof: We need to translate the string $D_{0} 0^{s}\left(D_{0}\right)^{k-1} 0^{s}\left(D_{1}\right)^{k-1} 0^{s}\left(D_{2}\right)^{k-1} 0^{s} \cdots$ from Lemma 2.12 into its generating function. Since

$$
\left|D_{0} 0^{s}\left(D_{0}\right)^{k-1} 0^{s} \cdots\left(D_{n-1}\right)^{k-1} 0^{s}\right|=s+1+\sum_{i=0}^{n-1}((k-1)[i+1]+s)=s+n(s-1)+[n+1],
$$

we can write

$$
\begin{aligned}
\mathcal{D}_{s}(z) & =z+\sum_{n \geq 0} z^{s+n(s-1)+[n+1]} D_{n}(z)\left(1+z^{\left|D_{n}\right|}+\cdots+z^{(k-2)\left|D_{n}\right|}\right) \\
& =z+\sum_{n \geq 0} \sum_{i=1}^{k-1} z^{s+n(s-1)+i[n+1]} D_{n}(z) \\
& =z+\sum_{n \geq 0} \sum_{i=1}^{k-1} z^{s+n(s-1)+i[n+1]+1} x_{1} x_{2} \cdots x_{n}
\end{aligned}
$$

where $x_{i}=z\left(1+z^{[i]}+\cdots+z^{(k-1)[i]}\right)=z[k]$. Recall that $D_{n}(z)=z x_{1} x_{2} \cdots x_{n}$ and expand the summation to obtain

$$
\begin{aligned}
\mathcal{D}_{s}(z) & =z+\left(z\left(z^{s+[1]}+\cdots+z^{s+(k-1)[1]}\right)\right)+\left(z x_{1}\left(z^{2 s-1+[2]}+\cdots+z^{2 s-1+(k-1)[2]}\right)\right)+\cdots \\
& =z\left(1+\left(z^{s+[1]}+\cdots+z^{s+(k-1)[1]}\right)+\left(x_{1}\left(z^{2 s-1+[2]}+\cdots+z^{2 s-1+(k-1)[2]}\right)\right)+\cdots\right. \\
& =z\left(1+z^{s+[1]}\left(\left(1+\cdots+z^{(k-2)[1]}\right)+\left(x_{1}\left(z^{s-1+k[1]}+\cdots+z^{s-1+(k-2)[2]+k[1]}\right)\right)+\cdots\right.\right. \\
& =z\left(1+z^{s+k^{0}}\left(\left(1+\cdots+z^{(k-2)[1]}\right)+z^{s-1+k[1]} x_{1}\left(\left(1+\cdots+z^{(k-2)[2]}\right)+\cdots\right.\right.\right. \\
& =z\left(1+z^{s+k^{0}}\left(\frac{1-z^{(k-1)[1]}}{1-z^{[1]}}+z^{s+k^{1}} \frac{1-z^{k[1]}}{1-z^{[1]}}\left(\frac{1-z^{(k-1)[2]}}{1-z^{[2]}}+z^{s+k^{2}} \frac{1-z^{k[2]}}{1-z^{[2]}} \cdots\right.\right.\right.
\end{aligned}
$$

The proofs of the next two theorems are omitted for space reasons. They will be included in the full paper.

Theorem 2.17 For all $s \geq 1$ and $k \geq 2$,

$$
\mathcal{A}_{s, k}(z)= \begin{cases}\frac{z}{1-z} \prod_{i \geq 1} \frac{1-z^{k[i]}}{1-z^{[i]}} & \text { if } s=0 \\ z \frac{1-z^{s}}{1-z} \sum_{n \geq 0} \prod_{i=1}^{n} z^{s} \frac{1-z^{k[i]}}{1-z^{[i]}} & \text { if } s>0 .\end{cases}
$$

Theorem 2.18 For all $s \geq 0$ and $k \geq 2$,

$$
\sum_{n \geq 0} p_{s}(n) z^{n}=\frac{1}{1-z}\left(z+z \sum_{m \geq 0} z^{k^{m}}\left(s+\frac{1}{1-z^{k^{m}}}\right)\right)
$$




\section{Compositions Of An Integer}

Jon Perry [12] has observed experimentally that $a_{1,2}(n)$ counts the number of compositions of $n$ such that, for some $m$,

$$
n_{0}+n_{1}+\cdots+n_{m}=n \text { where } n_{i} \in\left\{1,2^{i}\right\} \text { for } i=0,1, \ldots, m .
$$

He uses a notation similar to $\langle 1\rangle+\langle 1,2\rangle+\langle 1,4\rangle+\langle 1,8\rangle+\cdots$ to denote the set of such compositions and notes that many other combinatorial objects are in one-to-one correspondence with similar composition rules [12]. We call these rules specifications. Jackson and Ruskey [9] showed that $a_{s, 2}(n)$ counted the number of compositions of $n$ with specification

$$
\langle 1,2, \ldots, s\rangle+\langle s, 2+s-1\rangle+\langle s, 4+s-1\rangle+\langle s, 8+s-1\rangle+\cdots .
$$

Corollary 3.1 For $s \geq 1$, the number of compositions of $n$ with specification

$$
\langle 1,2, \ldots, s\rangle+\langle s, s+[1], \ldots, s+(k-1)[1]\rangle+\langle s, s+[2], \ldots, s+(k-1)[2]\rangle+\cdots
$$

is $a_{s, k}(n)$.

Proof: This is clear from the generating function of $\mathcal{A}_{s, k}(z)$ given in Theorem 2.17 by observing that $z\left(1-z^{s}\right) /(1-z)=\left(z+z^{2}+\cdots+z^{s}\right)$. Substituting into our summation gives

$$
\left(z+z^{2}+\cdots+z^{s}\right)\left(1+z^{s}\left(1+z^{[1]}+\cdots+z^{k[1]}\right)+z^{s}\left(1+\cdots+z^{k[1]}\right) z^{s}\left(1+\cdots+z^{k[2]}\right)+\cdots\right.
$$

As an example, $a_{2,3}(20)=10$, and the 10 corresponding compositions are given below:

$1+2+2+15, \quad 1+3+2+2+2+2+2+2+2+2, \quad 1+3+6+2+2+2+2+2, \quad 1+3+10+2+2+2$,

$1+4+15,2+2+2+2+2+2+2+2+2+2, \quad 2+2+6+2+2+2+2+2$,

$2+2+10+2+2+2, \quad 2+4+6+2+2+2+2, \quad 2+4+2+2+2+2+2+2+2$

\section{Acknowledgements}

We wish to thank Brad Jackson for helpful comments related to this research. We also wish to thank one of the referees for their very careful reading of the paper, which lead to many improvements in the presentation.

\section{References}

[1] J.-P. Allouche, J. Betrema, and J.O. Shallit, Sur des points fixes de morphismes d'un monoïde libre, Informatique théorique et Applications, 23 (1989) 235-249.

[2] Joseph Callaghan, John J. Chew III, and Stephen M. Tanny, On the behavior of a family of metaFibonacci sequences, SIAM J. Discrete Mathematics 18 (2005) 794-824.

[3] Peter J. Cameron, Combinatorics: topics, Techniques, Algorithms, Cambridge University Press, 1994.

[4] B.W. Conolly, Meta-Fibonacci sequences, Chapter XII in S. Vajda, Fibonacci \& Lucas Numbers, and the Golden Section, Ellis Horwood Limited, 1989.

[5] P. Flajolet and H. Prodinger, Level number sequences for trees, Discrete Mathematics 65 (1987) 149156.

[6] R.L. Graham, D.E. Knuth, and O. Patashnik, Concrete Mathematics, Addison-Wesley, second edition, 1994.

[7] R.K. Guy, Unsolved Problems in Number Theory, Problem Books in Math., Springer, New York, 1981.

[8] J. Higham and S. Tanny, More well-behaved meta-Fibonacci sequences, Congressus Numerantium, 98 (1993) 3-17.

[9] B. Jackson and F. Ruskey, Meta-Fibonacci sequences, binary trees and extremal compact codes, Electronic Journal of Combinatorics, 13 (2006), \#R26, 13 pages. 
[10] M. Khosravifard, M. Esmaeili, H. Saidi, and T.A. Gulliver, A tree based algorithm for generating all possible binary compact codes with $N$ codewords, IEICE Transactions on Fundamentals of Electronics, Communications and Computer Sciences, Vol. E86-A (2003) 2510-2516.

[11] D.E. Knuth, The Art of Computer Programming, Volume 1: Fundamental Algorithms, AddisonWesley, 1968.

[12] Jon Perry, Symmetric Ferrar Diagrams, website, www.users.globalnet.co.uk/ perry/maths/symmetricferrars/symmetricferrars.htm, March 2005.

[13] K. Pinn, Order and chaos in Hofstadter's $Q(n)$ sequence, Complexity 4 (1999) 41-46.

[14] S.M. Tanny, A well-behaved cousin of the Hofstadter sequence, Discrete Mathematics, 105 (1992) $227-239$. 
